\title{
A Framework for the Mixed Integer Dynamic Optimisation of Waste Water Treatment Plants using Scenario-Dependent Optimal Control
}

\author{
Jan Busch $^{\mathrm{a}}$, Marcella Santos ${ }^{\mathrm{b}}$, Jan Oldenburg ${ }^{\mathrm{c}}$, Andreas Cruse ${ }^{\mathrm{d}}$ and \\ Wolfgang Marquardt $\mathrm{a}^{\mathrm{a}^{*}}$ \\ ${ }^{a}$ Lehrstuhl für Prozesstechnik, RWTH Aachen, D-52056 Aachen, Germany \\ ${ }^{\mathrm{b}}$ School of Chemical Engineering, UNICAMP, Campinas - SP - Brazil \\ ${ }^{\mathrm{c}}$ BASF Aktiengesellschaft, D-67056 Ludwigshafen \\ ${ }^{\mathrm{d}}$ Uhde GmbH, Friedrich-Uhde-Str. 15, D-44141 Dortmund
}

\begin{abstract}
In many real life processes, operational objectives, constraints and the process itself may change over time. This is due to changing product requirements, market demands and other external or internal influences, which constitute a certain scenario. Modelbased techniques can provide optimal solutions to the corresponding scheduling and process control problems. This paper focuses on those situations, where the objectives and constraints of plant operation depend on the scenario and therefore change over time. A framework is developed, which enables the modelling and scheduling of different operational strategies on the optimisation horizon. A case study involving a waste water treatment plant is used to demonstrate the approach. Existing expert knowledge is used to relate certain operational objectives and constraints to corresponding scenarios. It is shown that easily interpretable optimisation results are obtained. Also, the results are significantly improved as compared to a weighted average approach only approximating sequential strategies.
\end{abstract}

Keywords: online optimisation, plant scheduling, scenario-dependent optimal control, waste water treatment, mixed integer dynamic optimisation

\section{Introduction}

With recent advances in the fields of process modelling, optimisation algorithms, computing power and practical experience, model-based techniques like online optimisation and plant scheduling have moved from being a purely academic challenge towards industrial relevance. Model-based control and scheduling require process models and models for the operational constraints and objectives. In the following, the operational constraints and objectives are defined as the operational strategy. Two typical operational strategies are to produce a certain product grade at minimum cost or at maximum throughput. Secondly, the scenario is defined to be the whole of those internal and ex-

*Author to whom correspondence should be addressed: marquardt@1pt.rwth-aachen.de 
ternal factors, which determine a suitable operational strategy, e.g. stock hold-ups, predicted market demand etc. This paper focuses on those situations, where the operational strategy depends on the present scenario, which may change over time. If such a change occurs on the time horizon which is considered in the optimisation, two or more periods (stages) with different operational strategies have to be scheduled in a way to yield optimal overall plant performance. Therefore, a framework will be proposed, which allows for the accurate modelling and scheduling of such problems, introducing the notion of scenario-dependent optimal control.
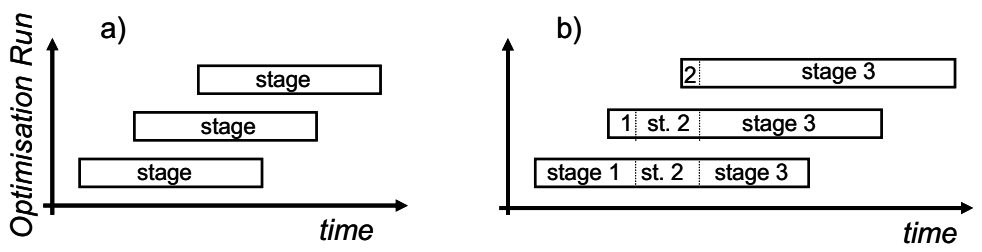

Figure 1a,b. Moving Horizon Control and Plant Scheduling

\section{State of the art}

The types of processes considered in this work are continuous processes, for which optimal base controller set point trajectories are to be determined using dynamic real time optimisation. Disturbances on a fast time scale are not accounted for on this control level, but long-term, predictable disturbances are considered. The optimisation is iteratively performed on a moving horizon, as depicted in Fig. 1a. In the following, two approaches will be introduced, which have been extensively treated in literature and which are valid for certain problem specifications. Based on this, the idea of scenariodependent optimal control will be developed.

If the operational objectives and constraints are assumed to be time invariant, the horizon can be interpreted as one stage, on which the continuous optimisation problem is solved (single stage formulation).

A plant scheduling problem evolves, when different products or product grades are to be produced sequentially, as e.g. in modern multipurpose plants (Papageorgiou et al., 1996). Here not only the individual production campaigns have to be optimised, but also their number, sequence and lengths. The optimisation horizon is split into stages, which correspond to the campaigns. On each stage, the process model and the operational constraints may be different. However, the operational objective is the same on all stages. Although usually performed offline, an iterative moving horizon implementation is shown in Fig. 1b.

\section{Scenario-dependent optimal control}

\subsection{Motivation}

In contrast to these approaches, this paper considers situations where the operational constraints and the objectives change over time. Abel and Marquardt (2000) have treated the case that at every point in time a critical event could take place, e.g. the burst of a rupture disc, after which it must still be possible to meet certain safety constraints 
using a suitable operational strategy. Since the moment of failure is expected at every point in time, this leads to a high problem complexity.

Here the case is considered that the sequence of operational strategies depends on predictable scenarios. The motivation to do so originates from research on the optimisation-based control of the waste water treatment plant discussed in the case study. When trying to formulate a suitable model-based operational strategy, it was found that plant operators employ two different strategies. At certain times the objective is to maximise the throughput in order to increase flexibility, at other times it is to minimise the energy consumption. The choice of a strategy mainly depends on the predicted inflow and therefore changes over time.

There are several possibilities to approximate the use of scenario-dependent operational strategies, e.g. by considering a weighted average of the individual objectives. This weighted average can be expressed in a single objective function, which is valid at all times and which can be optimised using a single stage formulation. The main difficulty is assigning suitable values to the weights, which means e.g. to assign a monetary value to flexibility that is valid in all possible scenarios. However, to the authors' knowledge, no exact modelling and scheduling methodology for scenario-dependent operational strategies has been reported so far.

This paper proposes such a rigorous approach, which can be interpreted as a variation of regular plant scheduling on a moving horizon. The campaigns are substituted by time periods, during which a certain operational strategy is employed. The main difference is that not only the constraints, but also the operational objectives may change from stage to stage, while the process model is always the same. The expected benefits of the are:

1. Expert knowledge concerning the strategies and the heuristics, when to employ which strategy, can be exactly represented. This might enhance the overall optimisation results as compared to approaches only approximating sequential strategies.

2. Since the optimisation results correspond to well known strategies, which are separated in time, they are more easily interpretable by plant operators. This is a key factor in enhancing the acceptance of model-based techniques in industrial settings.

\subsection{Formulation of the optimisation problem}

$$
\begin{array}{lll}
\min _{\boldsymbol{u}, t_{f, k}} \sum_{k=1}^{K} w_{k} \phi_{k}\left(\boldsymbol{x}\left(t_{f, k}\right), \boldsymbol{z}\left(t_{f, k}\right), \boldsymbol{u}\left(t_{f, k}\right), \boldsymbol{d}\left(t_{f, k}\right), \boldsymbol{p}, t_{f, k}\right) & \\
& \boldsymbol{f}(\dot{\boldsymbol{x}}, \boldsymbol{x}, \boldsymbol{z}, \boldsymbol{u}, \boldsymbol{d}, \boldsymbol{p}, t)=\boldsymbol{0}, \boldsymbol{x}\left(t_{0,1}\right)=\boldsymbol{x}_{0}, t \in\left[t_{0, k}, t_{f, k}\right], & \\
& \boldsymbol{g}^{*}(\dot{\boldsymbol{x}}, \boldsymbol{x}, \boldsymbol{z}, \boldsymbol{u}, \boldsymbol{d}, \boldsymbol{p}, t) \leq \boldsymbol{0}, & \\
& \boldsymbol{g}_{k}(\dot{\boldsymbol{x}}, \boldsymbol{x}, \boldsymbol{z}, \boldsymbol{u}, \boldsymbol{d}, \boldsymbol{p}, t) \leq \boldsymbol{0}, & k \in\{1, \ldots, K\}, \\
s . t . & \varphi_{s}\left(\dot{\boldsymbol{x}}\left(t_{f, s}\right), \boldsymbol{x}\left(t_{f, s}\right), \boldsymbol{z}\left(t_{f, s}\right), \boldsymbol{u}\left(t_{f, s}\right), \boldsymbol{d}\left(t_{f, s}\right), \boldsymbol{p}, t_{f, s}\right)=\boldsymbol{0}, & s \in\{1, \ldots, K-1\}, \\
& \boldsymbol{h}_{k_{s}}\left(\dot{\boldsymbol{x}}\left(t_{f, s}\right), \boldsymbol{x}\left(t_{f, s}\right), \boldsymbol{z}\left(t_{f, s}\right), \boldsymbol{u}\left(t_{f, s}\right), \boldsymbol{d}\left(t_{f, s}\right), \boldsymbol{p}, t_{f, s}\right) \leq \boldsymbol{0}, & s \in\{1, \ldots, K-1\}, \\
& \boldsymbol{\Gamma}\left(\boldsymbol{x}\left(t_{f, s}\right)\right)=\boldsymbol{x}\left(t_{0, s+1}\right), & s \in\{1, \ldots, K-1\} .
\end{array}
$$

The proposed problem formulation is given by ( $\mathrm{P} 1)$. The process model, which is valid on all stages, is determined by $\boldsymbol{f}$ and $\boldsymbol{g}^{*}$. The disturbances are given by $\boldsymbol{d}, \boldsymbol{u}$ are the controls, $\boldsymbol{x}$ are the differential and $\boldsymbol{z}$ are the algebraic state variables, and $\boldsymbol{p}$ are the parame- 
ters. The sequence of operational strategies is reflected by a sequence of $K$ stages on the optimisation horizon. Each stage $k$ is assigned one operational strategy, which is modelled in detail by a set of constraints $\boldsymbol{g}_{k}$ and an objective function $\phi_{k}$. The length of stage $k$ is defined by the interval $\left[t_{0, k}, t_{f, k}\right]$. Between the stages, $K$-1 switching conditions $\varphi_{s}$ and $\boldsymbol{h}_{s}$ and stage transition conditions $\boldsymbol{\Gamma}_{s}$ for the differential states have to be defined. The initial conditions $\boldsymbol{x}_{0}$ have to be determined from measurements or from state estimation. In the overall objective function, the weighted objective functions of the stages are summed up. For two reasons, these weights are much easier to choose than in weighted average approaches. In case there is only one strategy used on the optimisation horizon, no weighting is considered at all. If several strategies are employed, the weighting only has the role of setting priorities among the objectives in a certain scenario, as will be demonstrated in the case study.

Since the number, type and order of strategies are not known beforehand, they represent discrete degrees of freedom. There are two possibilities to determine the values for the discrete variables: either they are determined by an appropriate optimisation algorithm together with the continuous optimisation problem, or they are determined beforehand by some decision algorithm or expert knowledge. If they can be determined beforehand, the problem reduces to a so-called multistage problem. Otherwise, a mixed integer dynamic optimisation (MIDO) problem has to be solved. Due to limited space, only the formulation of the multistage problem is presented here. The formulation of the MIDO problem can be derived from Oldenburg et al. (2003).

Multistage and especially MIDO problems are computationally more demanding than single stage problems. However, since planning problems are usually solved on longer time horizons, the increase in computation time is not expected to be a limiting factor. The software tool DyOS (DyOS, 2002), which has been developed at the LPT, is an efficient tool for solving problems of type (P1) as well as MIDO problems and is used in the following case study (Schlegel et al., 2004).

\section{Case study: Waste water treatment plant}

The proposed methodology will be exemplified in a case study involving a simulated municipal waste water treatment plant (WWTP). A simplified flowsheet of the process is shown in Fig. 2. Incoming waste water is stored in a buffer tank. From there, it is transferred to a biological stage, consisting of a denitrification and a nitrification tank, which are connected by a recycle stream. Air can be blown into the nitrification tank and sludge can be withdrawn from it. The biomass and the purified water (product) are separated by membrane filtration. The relevant operational constraints are the upper limits of the buffer tank volume and of the permeate ammonia concentration. The operational cost of the process is mainly caused by the air flow. There is a maximum power consumption level, above which the cost increases dramatically. In the following, this is related to an air flow threshold. From discussions with plant operators, it is understood that there are two operational strategies, whose employment mainly depends on the predicted inflow:

1. Regular inflow: The aim is to bring the buffer tank volume to a certain, usually low target value, while keeping the air flow below the threshold. This is called the flexibility strategy, as it increases flexibility for unforeseen high inflow situations. 
2. High inflow: If staying below the air flow threshold and meeting all constraints cannot be achieved due to high inflow, the air flow is allowed to exceed the threshold, but as little as possible. This is called the economics strategy.

These two strategies are modelled with appropriate constraints and objective functions. The stage transition conditions $\Gamma$ enforce continuity of all $\boldsymbol{x}$ between all stages. No switching conditions $\boldsymbol{h}$ or $\varphi$ are required. The process model $\boldsymbol{f}, \boldsymbol{g}^{*}$ is given by biological kinetics (Gujer et al., 1999) and mass balances. The membrane is assumed to be an ideal splitter. The resulting model consists of 42 differential and 236 algebraic equations. The size of the problem mainly results from the 13 components considered in each tank. The overall length of the optimisation horizon is 3 days, for which a perfect inflow prediction is assumed to be available. In this case study, a new optimisation run is performed once per day. The sequence of operational strategies on the horizon is determined manually by inspection of the predicted inflow. The weights $w_{k}$ are chosen such that the minimisation of cost has priority over the increase of flexibility. The degrees of freedom are the controls $\boldsymbol{u}$ and the endpoints of the stages $t_{f, s}$.

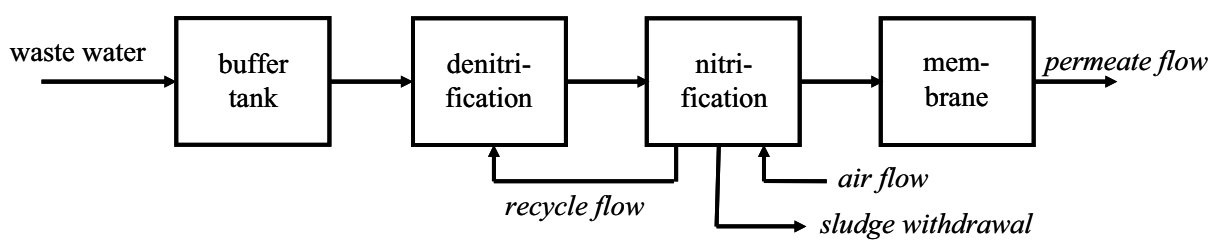

Figure 2. Schematic representation of the waste water treatment plant

Some results of an extensive optimisation study are depicted in Fig. 3. The inflow history is characterised by a strong rain event on day 7 and a smaller one on day 10 , superposed by the regular daily inflow. From day 1 to day 4, the flexibility strategy is applied. This implies that the air flow does not exceed the threshold of $551 /$ day and that the objective of the optimisation is to lower the volume in the buffer tank. On day 5, the strong rain event on day 7 is predicted. As now the flexibility strategy would become infeasible, the economics strategy is employed. The air flow increases as much as necessary. This in turn allows the permeate flow to increase such that the upper limit of the buffer tank volume of $3000 \mathrm{~m}^{3}$ is met. At the end of day 7, the flexibility strategy is employed again.

The interpretation of the results is straightforward, because at every point in time only one operational strategy is employed, the choice of which is deduced from the operational context. This enables simple relations between the operational objective and constraints and the resulting input and state trajectories.

Fig. 3 also shows the computed trajectories in the same setting, but approximating the problem with a weighted average single stage formulation. The deviation from the air flow threshold and from the buffer volume target value are weighted and summed up in a single objective function. This implies that both objectives are considered at every point in time. Several weights have been tried and the most successful combination is presented here. While the computed permeate flow and the resulting buffer tank volume are very similar to the results of the multistage optimisation, a different recycle stream and a much higher air flow are computed, leading to an increase of cost by a factor of $170 \%$. This shows that separating the strategies and sequencing them, as suggested by 
practitioners, leads to substantially better optimisation results. Also, the interpretation of the single stage results is not straightforward (consider e.g. the peak of the air flow on day 7), since at every point in time two competing objectives are considered.
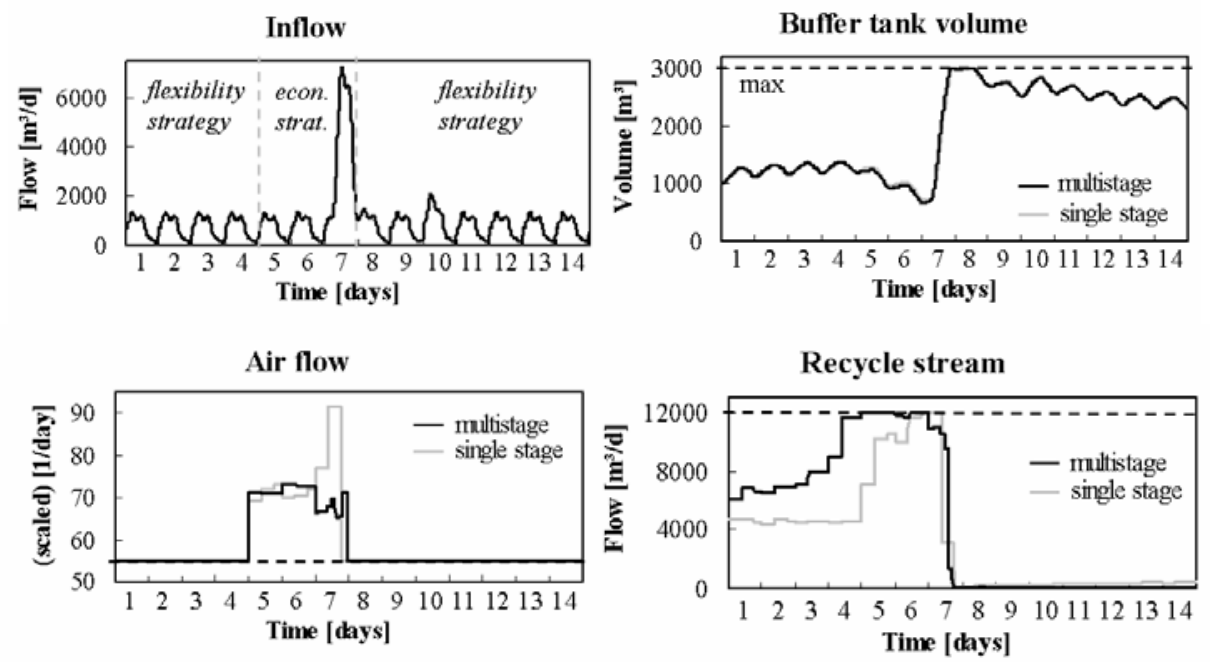

Figure 3. Optimisation results (multistage and single stage formulation)

\section{Conclusions}

Scenario-dependent optimal control allows to model and schedule sequences of different operational strategies, each of which is employed in a certain operational scenario. It leads to easily interpretable and, in the presented case study, to significantly improved optimisation results. Future research should analyse for which processes improved optimisation results can be expected and concentrate on applications. Reliable disturbance forecasting and automatic sequencing are important aspects in this context.

\section{References}

Abel, O., Marquardt, W., 2000, Scenario-Integrated Modeling and Optimization of Dynamic Systems, AIChE Journal 46, 4.

DyOS, 2002, DyOS User Manual, Release 2.1, Lehrstuhl für Prozesstechnik, RWTH Aachen, Aachen.

Gujer, W., Henze, M., Mino, T. and van Loosdrecht, M., 1999, Activated Sludge Model No. 3, Water Science and Technology 39, 1.

Oldenburg, J., Marquardt, W., Heinz, D., Leineweber, D. B., 2003, Mixed-Logic Dynamic Optimization Applied to Batch Distillation Process Design, AIChE Journal 49, 11.

Papageorgiou, L. G., Pantelides, C. C., 1996, Optimal campaign planning scheduling of multipurpose batch semicontinuous plants. 1. Mathematical formulation, Ind. Eng. Chem. Res. 35, 2.

Schlegel, M., Stockmann, K., Binder, T., Marquardt, W., 2004, Dynamic optimization using adaptive control vector parameterization, submitted to: Computers \& Chemical Engineering.

\section{Acknowledgements}

The financial support by the DFG (German Research Foundation) in the project "Optimisation-based process control of chemical processes" is gratefully acknowledged. 